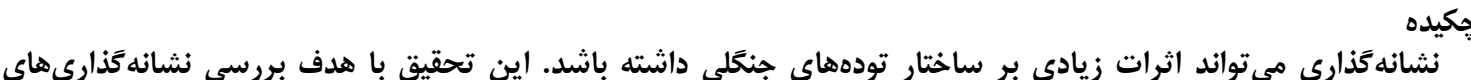

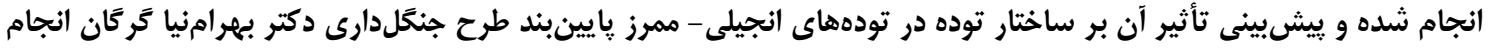

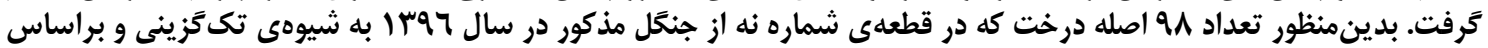

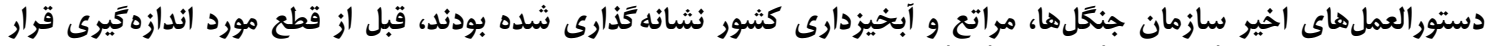

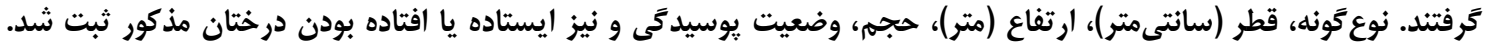

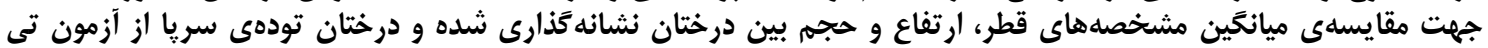

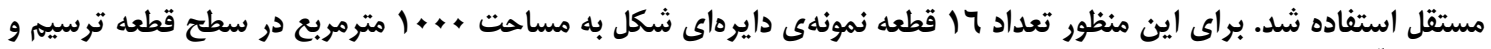

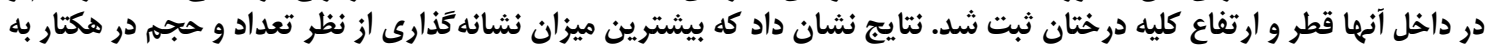

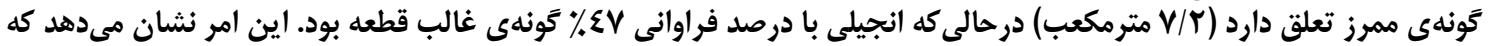

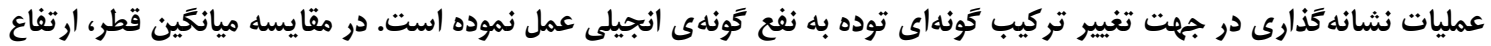

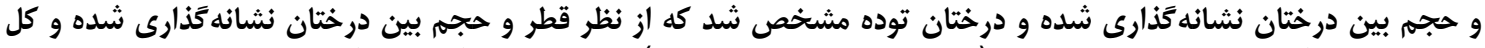

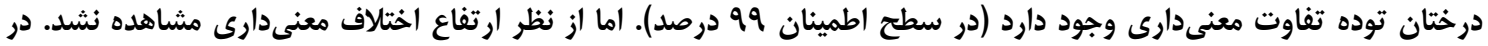

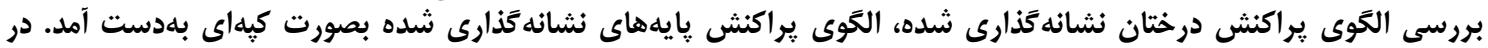

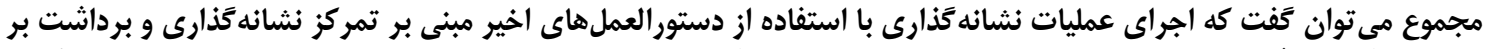

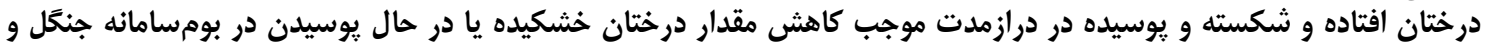
كاهش توان بومشناختى اين بومسامانه مىشود دورد.

وازههاى كليدى: جنكَلشناسى، ساختار، شيوهى تكَّزينى، خردز يستَاه، الحَوى يراكنش

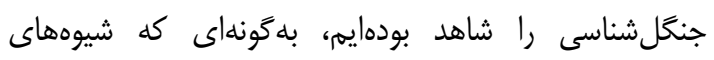

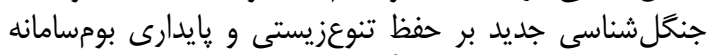

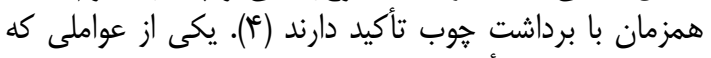

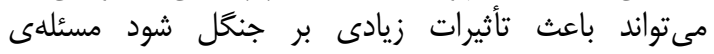

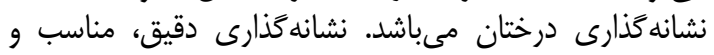

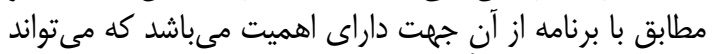

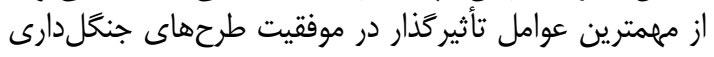

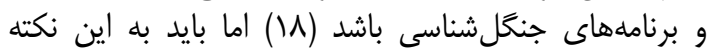

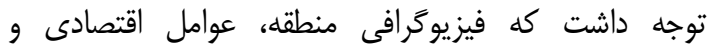

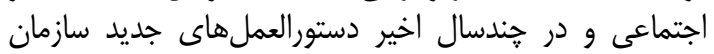

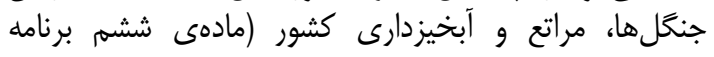

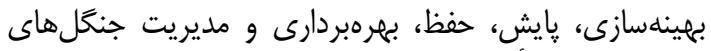

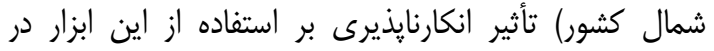

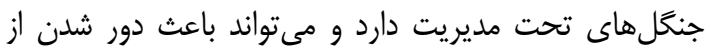

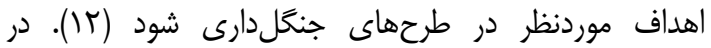
دستورالعملهاى اخيرسازمان جنغلالها، مراتع و و آبخيزدارى

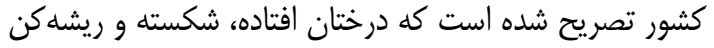

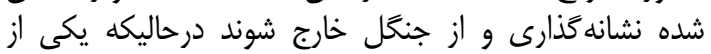

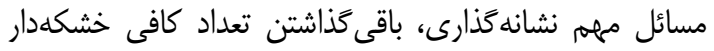

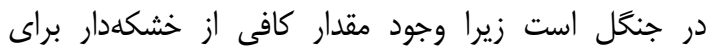

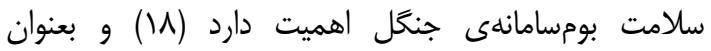
زيستخاهى براى بسيارى از موجودات زنده نقش مهام الهمى در

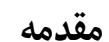

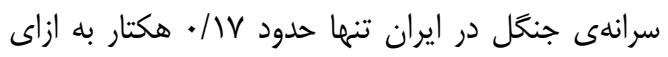

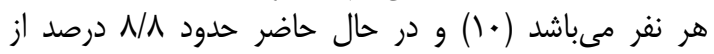

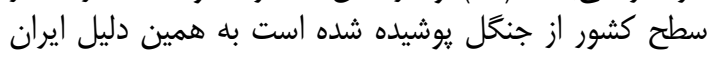

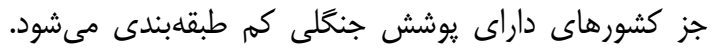

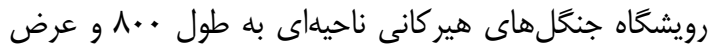

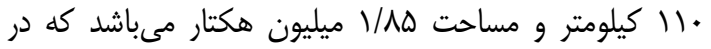

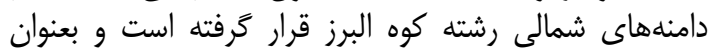

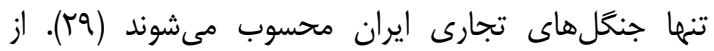

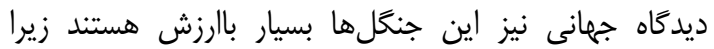

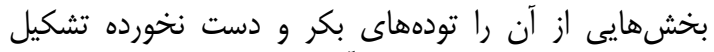

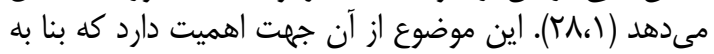

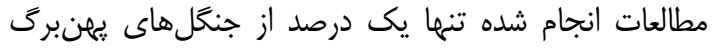

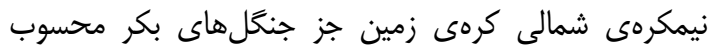

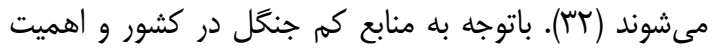

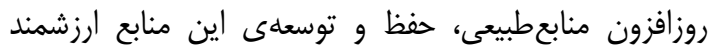

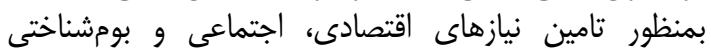

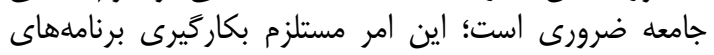

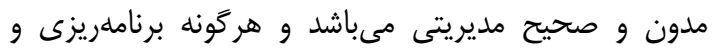

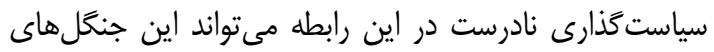

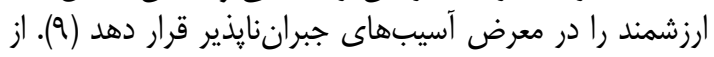
اينروست كه همواره طى ساليان كذنشته تكامل شيوههاى 


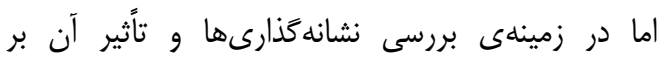

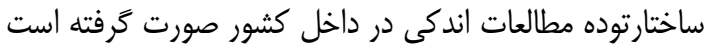

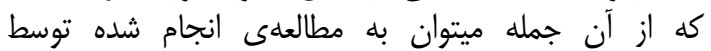

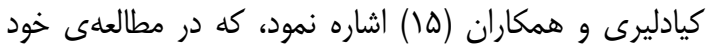

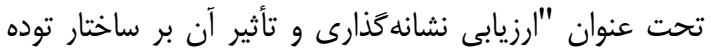

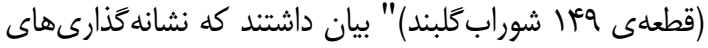

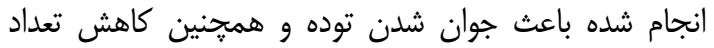

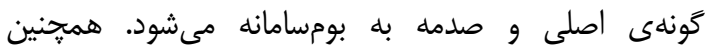

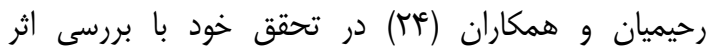

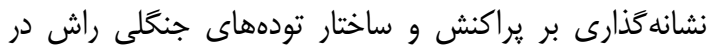

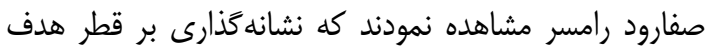

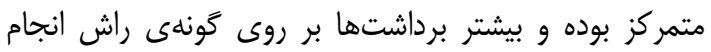

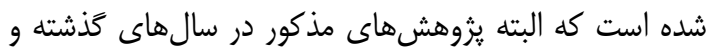

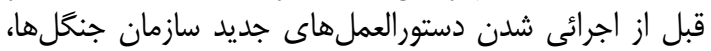

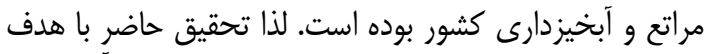

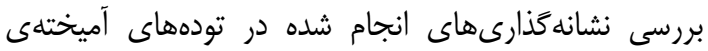

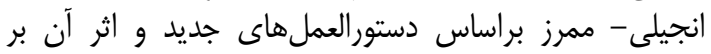
ساختار توده انجام شده است.

\section{مواد و روشها}

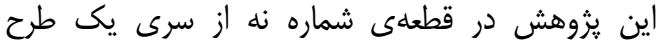

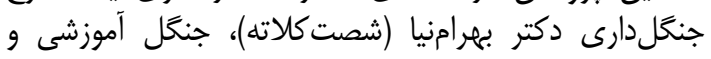

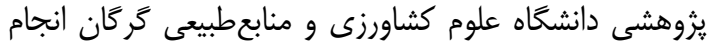

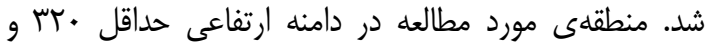

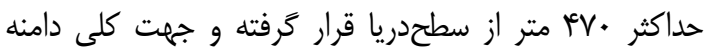

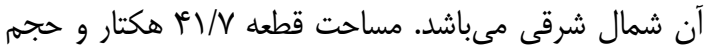

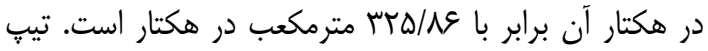

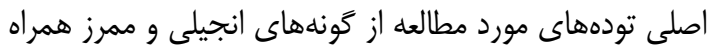

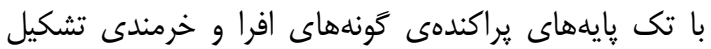

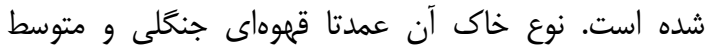

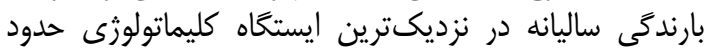

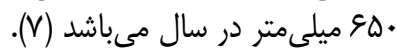

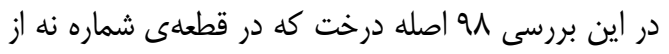

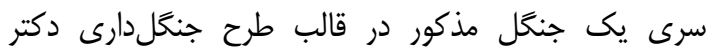

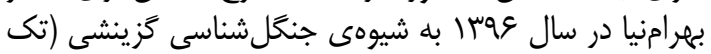

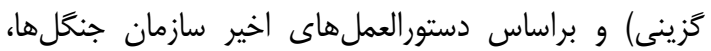

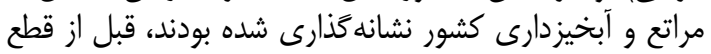

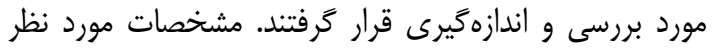

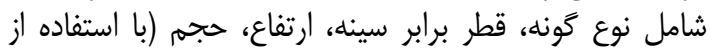

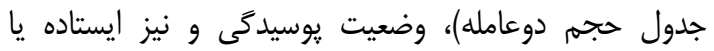

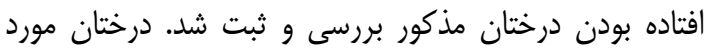

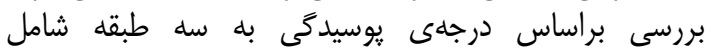

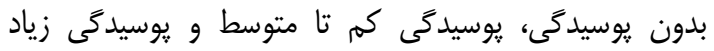

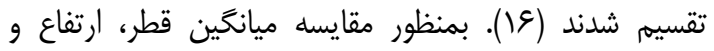

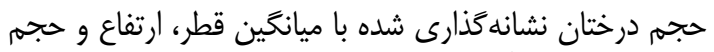

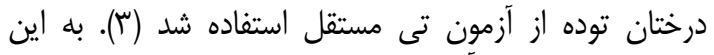

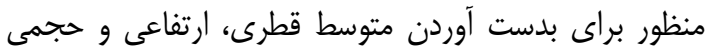

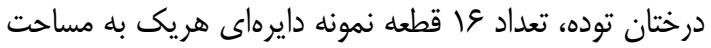
ده آر ( .... مترمربع) بصورت سيستماتيك تصادفى و با ابعاد

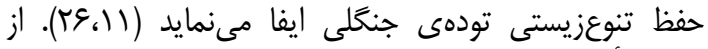

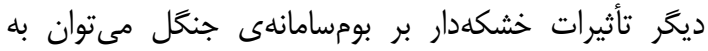

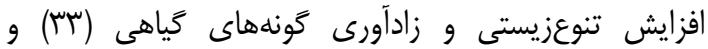

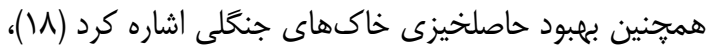

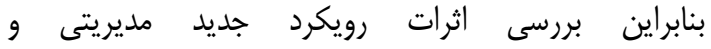

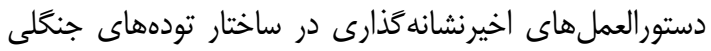
حائز اهميت بنظر مىرسل.

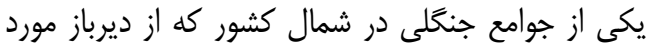

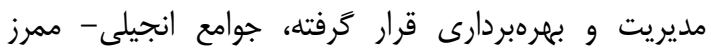

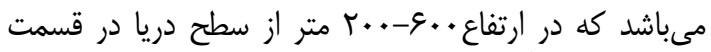

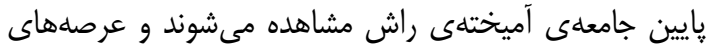

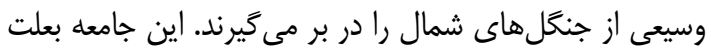

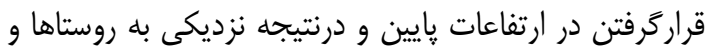

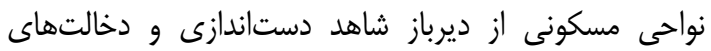

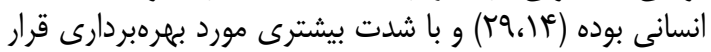

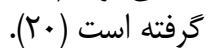
تاكنون مطالعات متعددى در رابطه با برات برسى اثرات

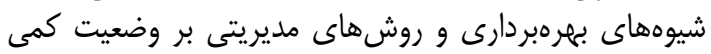

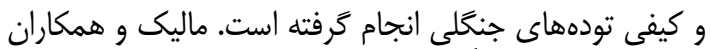
نئ (IV)

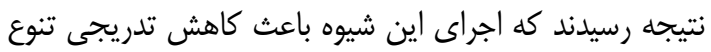

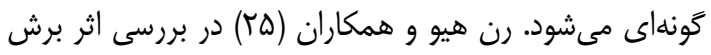

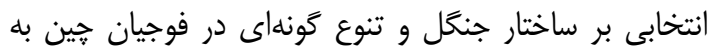

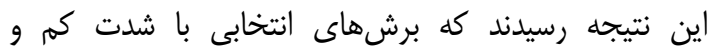

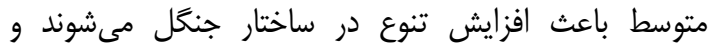

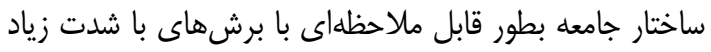

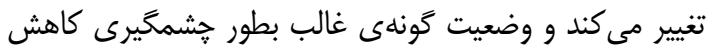

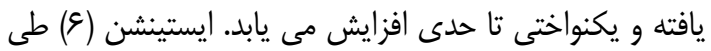

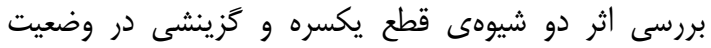

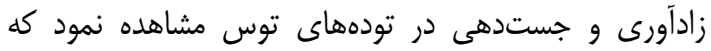

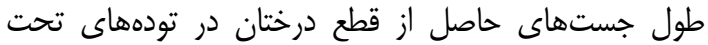

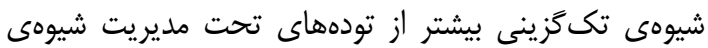

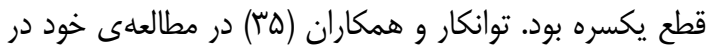

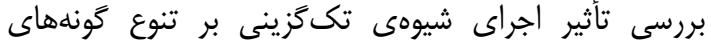

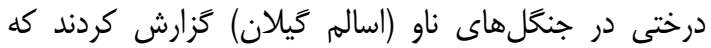

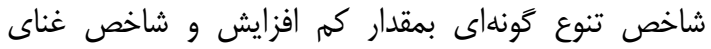

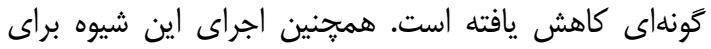

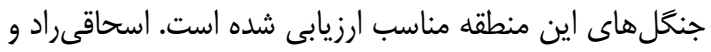

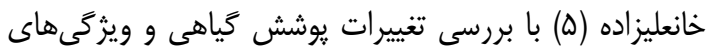

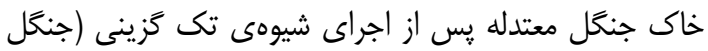

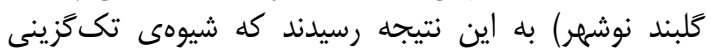

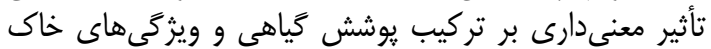
در قطعهى مديريت شده در مقابل قطعهى ترى شاهد نداشته است.

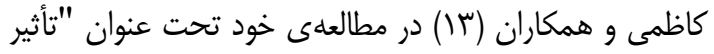

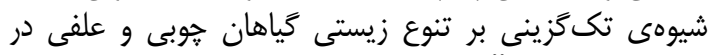

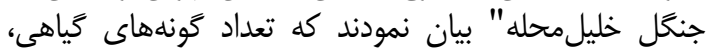

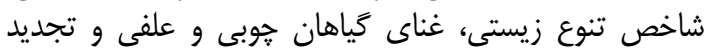

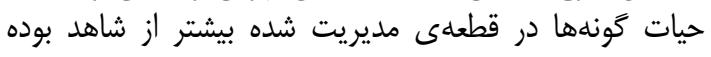


حالى كه E (r) مقدار اين فاصله در يك جنكل با توزيع كاملا

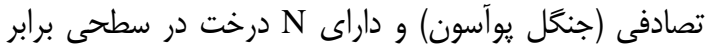

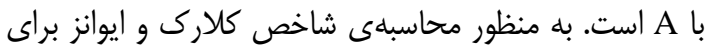

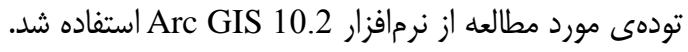

\section{نتايج و بحث}

نتايج بررسى درختان نشانه كذارى شده در قطعهى شماره

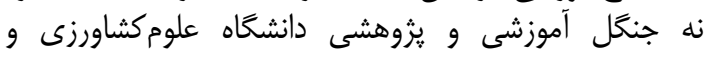

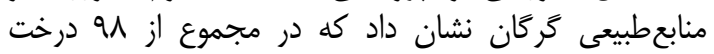

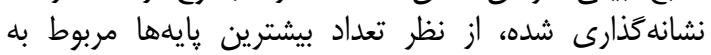

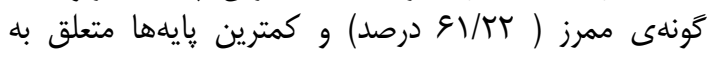

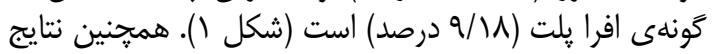

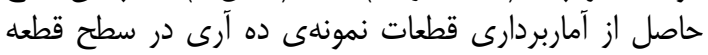

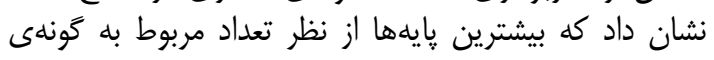

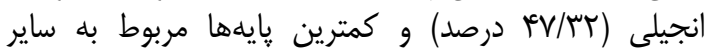

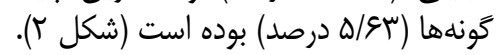
حداقل قطر درختان نشانه كذارى شده شاه

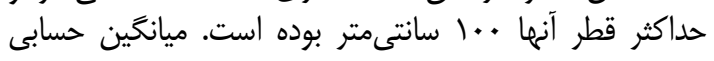

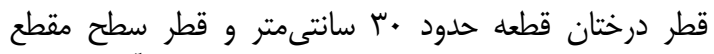

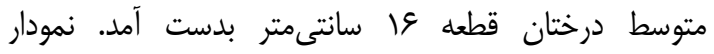

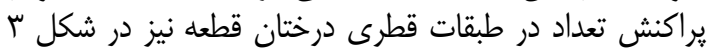
نشان داده شده است.

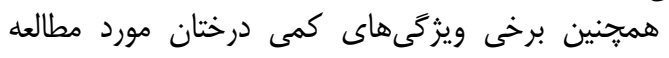
بترتيب شامل حجم، قطر و ارتفاع در جدول انشئ درخان داده شده
شبكه • •Y × • ا متر در سطح قطعه ترسيم شده و در داخل

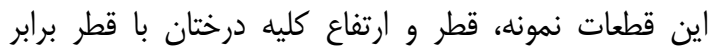

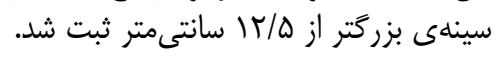

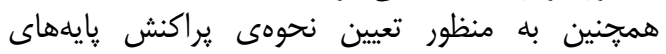

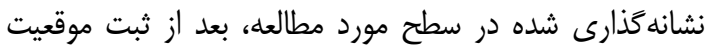

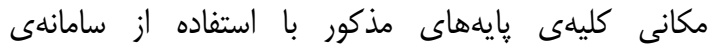
موقعيتياب جهانى، از شاخص كلاركى و ايوانز (رابطه الماني )

$\boldsymbol{E}(\boldsymbol{r})=\frac{1}{2 \times \sqrt{\frac{N}{A}}}$

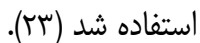

$$
C E=\frac{r_{A}}{r_{E}} \quad r_{E}=\frac{1}{2 \sqrt{\frac{N}{A}}}
$$

در اين رابطه A: مساحت تودمى جنكلى و N: تعداد درختان مورد مطالعه است. در رابطهى فوق متوسط فاصله بين درختان با فاصلهى

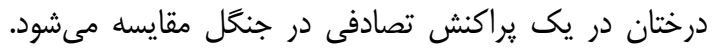

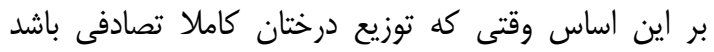

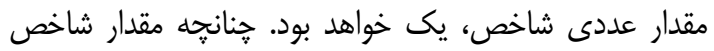

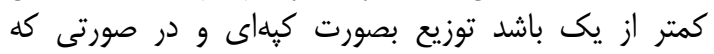

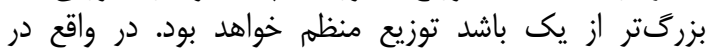

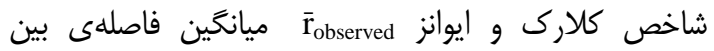

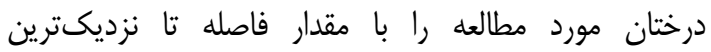

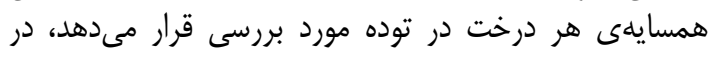

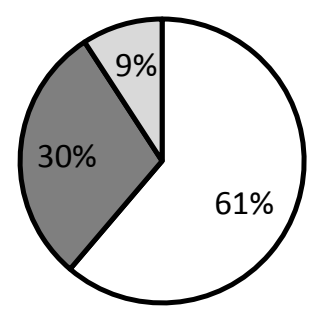
Dمرز
انجيلى
(افرا

Carpinus betulus Parrotia persica Acer velutinum 


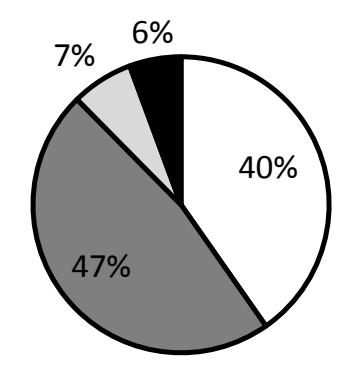
口مرز
انجيلى
|فرا
ساير

Carpinus betulus Parrotia persica Acer velutinum Other species

شكل r- درصد فراوانى درختان موجود در قطعات نمونه ده آرى (درصد)

Figure 2. Species composition of trees in 1000 square meter sample plots (\%)

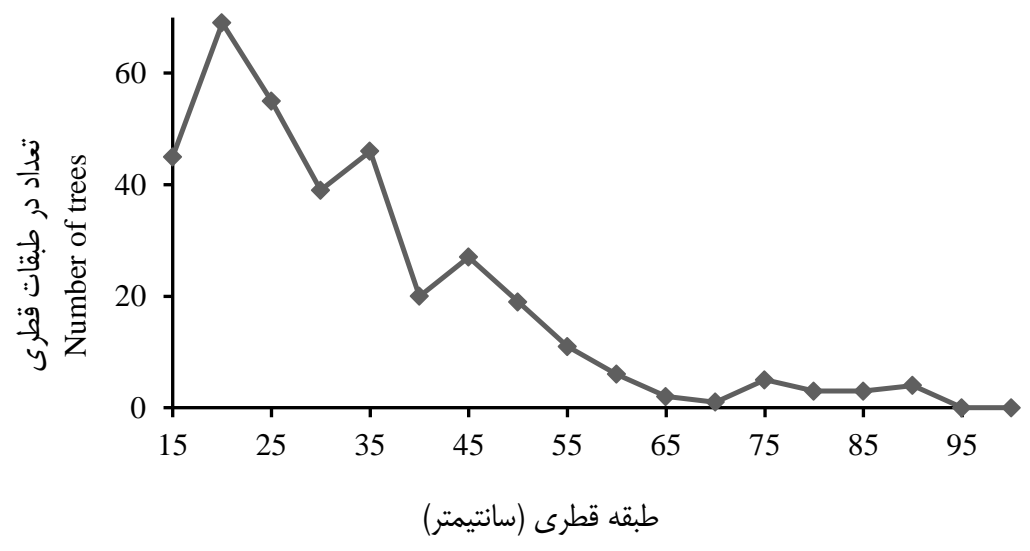

DBH classes (cm)

شكل r- ير اكنش تعداد در طبقات قطرى قطعه

Figure 3. Diameter distribution in compartment 
جدول (- كمينه، بيشينه و ميانكَين حجم، قطر و ارتفاع درختان نشانه كَارى شده و درختان كل قطعه Table 1. Min, max and mean of volume, diameter and height of marked trees and whole of the compartment trees

\begin{tabular}{|c|c|c|c|c|c|c|c|c|c|c|c|}
\hline & $\begin{array}{l}\text { ميانگيn } \\
\text { Mean }\end{array}$ & & & $\begin{array}{l}\text { Max. } \\
\text { Maشنd }\end{array}$ & & & $\begin{array}{l}\text { كمينd } \\
\text { Min. }\end{array}$ & & \multirow{2}{*}{ Species } & \multirow[b]{2}{*}{ كونه } & \\
\hline $\begin{array}{c}\text { ارتفاع } \\
\text { Height (m) } \\
\text { Heige }\end{array}$ & $\begin{array}{c}\text { قطر } \\
\text { (سانتى (cm) } \\
\text { Diameter (cm) }\end{array}$ & $\begin{array}{c}\text { حجم) } \\
\text { (مترمكب) } \\
\text { Volume }\left(m^{3}\right)\end{array}$ & $\begin{array}{c}\text { ارتفاع } \\
\text { height (m) } \\
\end{array}$ & $\begin{array}{l}\text { قطر (سانتىمتر) } \\
\text { Diameter (cm) }\end{array}$ & $\begin{array}{c}\text { حجمب (مترمكب) } \\
\text { Volume }\left(m^{3}\right) \\
\end{array}$ & $\begin{array}{c}\text { ارتفاع } \\
\text { (متر) } \\
\text { Height (m) }\end{array}$ & $\begin{array}{c}\text { قطر } \\
\text { (سانتىمتر) } \\
\text { Diameter (cm) }\end{array}$ & حجم (مترمكعب) & & & \\
\hline$r M / \cdot r$ & $g r / A \mu$ & $\Delta / \cdot V$ & rr & 90 & 1.1 .1 & D/IT & rQ & $\cdot / \Delta T$ & Carpinus betulus $\mathrm{L}$. & ممرز & \\
\hline$|V / 4|$ & 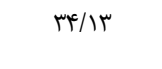 &.$/ 98$ & $r / r$ & VQ & $r / 9 \Lambda$ & 11 & ra & . & Parrotia Persica (DC.) C.A.Mey. & انجيلى & درختان نشانهكذارى شده \\
\hline $19 / \pi$ & $\Delta q / \overline{|c|}$ & $r / v e$ & $r N / \Delta$ & $1 .$. & $9 / 4 \wedge$ & 9 & $r$. & $\cdot / 9 V$ & Acer velutinum Boiss. & افرا & \\
\hline ת א & 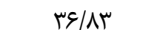 & $1 / \Delta \omega$ & $r \Delta / 1$ & q. & $q / ז \Delta$ & $9 / 4$ & rו & .1 .9 & Carpinus betulus $\mathrm{L}$. & مرز & \\
\hline$|\omega / V|$ & $r \Delta / q)$ & $\cdot / \Delta \Delta$ & tr & vr & r/Tr & $8 / \Delta$ & $\mid r / \Delta$ & $.1 \cdot 4$ & Parrotia Persica (DC.) C.A.Mey. & انجيلى & درختان قطعه \\
\hline $1 / / 9$ & $r q / 8$ & $\cdot / \Lambda t$ & $T V / Q$ & $\Delta F$ & $r / N$ & 1.11 & $\mid r / \Delta$ & .1 .9 & Acer velutinum Boiss. & افرا & Trees of compartment \\
\hline$r \cdot / 4 e^{2}$ & $r / / v$ &.$/ 98$ & $T V / T$ & $\Delta \wedge$ & $r / \cdot r$ & $N / c^{c}$ & $\mid r / \Delta$ & .1 .9 & Other species & ساير" & \\
\hline & & & & & & & & & & & * *: شيردار و خرمندى \\
\hline
\end{tabular}




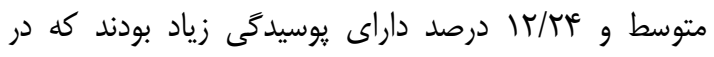

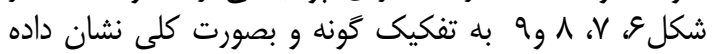
شده است.

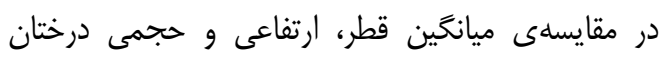

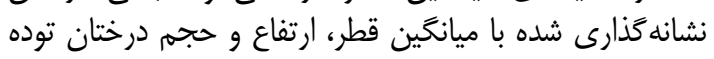

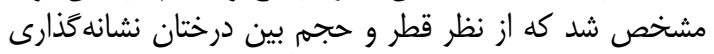

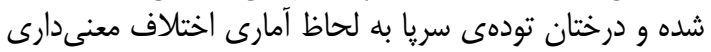

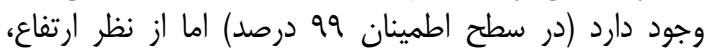

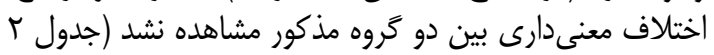

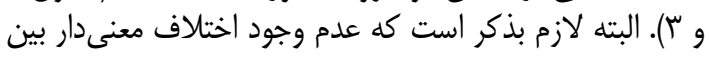

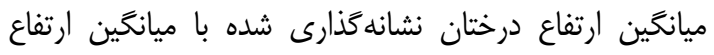

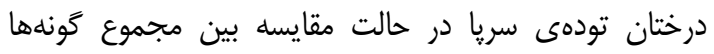

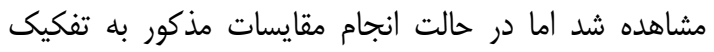

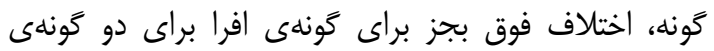

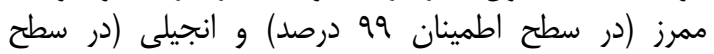
اطمينان Q9 درصد) معنىدار بود.

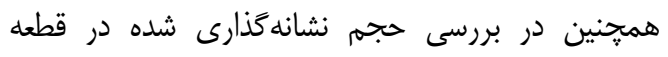

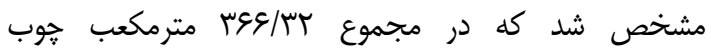

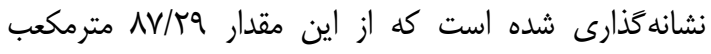

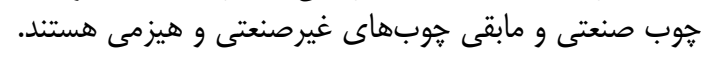

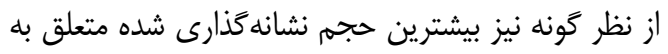

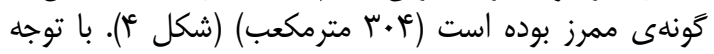

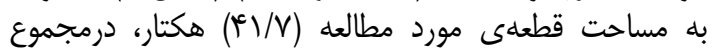

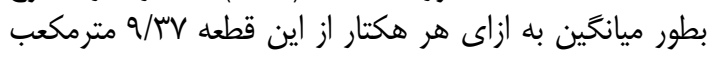
جوب نشانه خذارى شده است.

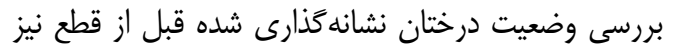

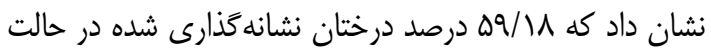

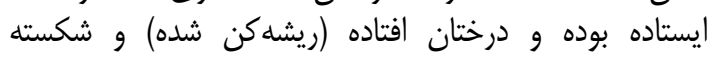

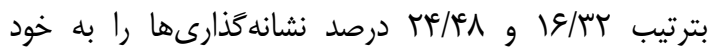

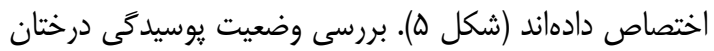

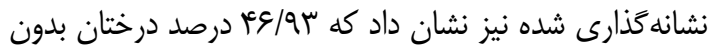

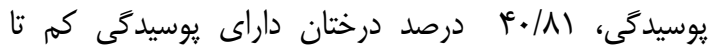


Table 2. Comparison of height (m) means by independent t-test

جدول r- مقايسهى ميانخَين ارتفاع (متر) با استفاده از آزمون تى مستقل

\begin{tabular}{|c|c|c|c|c|c|c|}
\hline خطاى معيار تفاوت ميانگ̌ين & $\begin{array}{c}\text { تفاوت ميانگ̌نen } \\
\text { Mean Difference }\end{array}$ & 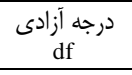 & $\mathrm{t}$ & $\begin{array}{c}\text { سطح معنى دارى } \\
\text { Sig. } \\
\end{array}$ & Species & كونه \\
\hline . /Ar & r/Le & $\Lambda r / \Lambda)$ & $r / N S$ &.$/ .1$ & Carpinus betulus $\mathrm{L}$. & ممرز از \\
\hline$\cdot / \mathrm{vA}$ & $-1 / V$ & 190 & $-Y / I V$ & | & Parrotia Persica (DC.) C.A.Mey. & انجيلى \\
\hline T/ & 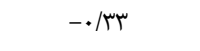 & r &.$- / 14$ & $\cdot / \mathrm{M}$ & Acer velutinum Boiss. & افرا \\
\hline سو/. & $-\cdot / \omega$ & 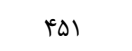 & $-\cdot / \wedge$ & Thtא. & Total & 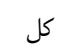 \\
\hline
\end{tabular}

Table 3. Comparison of diameter $(\mathrm{cm})$ and volume $\left(\mathrm{m}^{3}\right)$ means by independent t-test

جدول بـ- مقايسهى ميانگَين قطر (سانتىمتر) و حجم (مترمكعب) با استفاده از آزمون تى مستقل

\begin{tabular}{|c|c|c|c|c|c|c|c|c|c|c|c|}
\hline \multicolumn{2}{|c|}{ خطاى معيار تفاوت ميانكَين } & \multicolumn{2}{|c|}{$\begin{array}{c}\text { تفاوت ميانگين } \\
\text { Mean Difference }\end{array}$} & \multicolumn{2}{|c|}{ 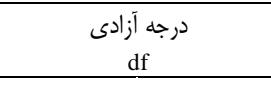 } & \multicolumn{2}{|c|}{$\mathrm{t}$} & \multicolumn{2}{|c|}{$\begin{array}{l}\text { سطح معنى دارى } \\
\text { Sig. }\end{array}$} & \multirow[t]{2}{*}{ Species } & \multirow[t]{2}{*}{ 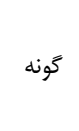 } \\
\hline حجم & قطر & حجم & قطر & حجم & قطر & حجم & قطر & حجم & قطر & & \\
\hline.$/ \% \Delta$ & $r r / r$ & $-r / \Delta r$ & -rه/৭q & $V \Delta / \Delta \omega$ & $9 \mathrm{~V} / 9 \mathrm{~F}$ & $-V / V$ & $-N$ & $\%$ &.$/ . .1$ & Carpinus betulus $\mathrm{L}$. & 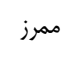 \\
\hline .119 & $r / r \Lambda$ & $-\cdot|+|$ & $-N / T r$ & $M E / F i$ & $r \cdot 190$ & $-r / r^{c}$ & $-r / \Delta$ & $/ \cdot .1$ & II & $\begin{array}{l}\text { Parrotia Persica (DC.) } \\
\text { C.A.Mey. }\end{array}$ & انجيلى \\
\hline $1 / \pi r$ & $9 / / \Delta$ & $-r / \cdot 1$ & $-r q / \Lambda e^{2}$ & 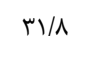 & $9 / v q$ & $-r / r$ & $-r / r$ & $\% \cdots$ & $\cdot / M$ & Acer velutinum Boiss. & افرا \\
\hline . I L & $r / \Delta T$ & $-r / V \Delta$ & תות & $1.9 / \pi$ & $\mid \pi r / 9$ & $-V / \Lambda$ & $-9 / 1$ & $\%$ & Trאוא. & Total & كل \\
\hline
\end{tabular}




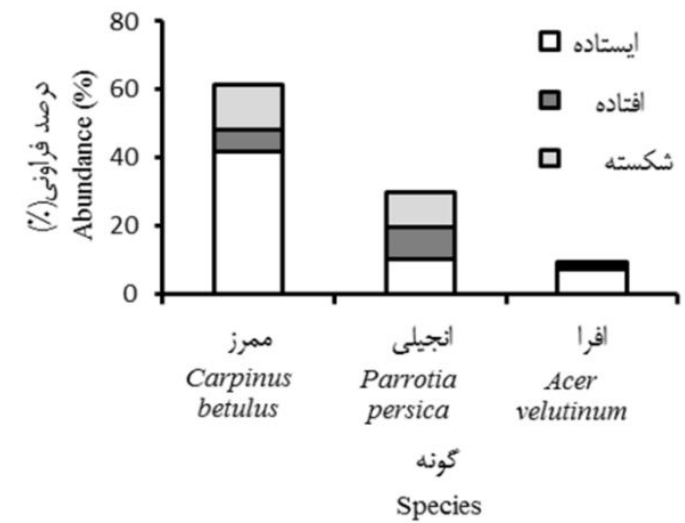

شكل ه- وضعيت ايستاده، افتاده و شكسته بودن درختان نشانه كَارى شده به تفكيك گونه

Figure 5. Abundance of standing, fallen and broken trees by species among marked tree

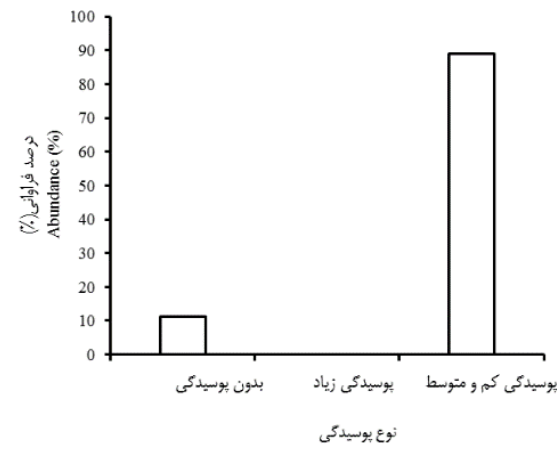

$$
\text { شكل V- نوع :وسيدگى پايههاى نشانه گذارى شده افرا }
$$

Figure 7. Severity and amount of decay of marked trees based on species Acer velutinum

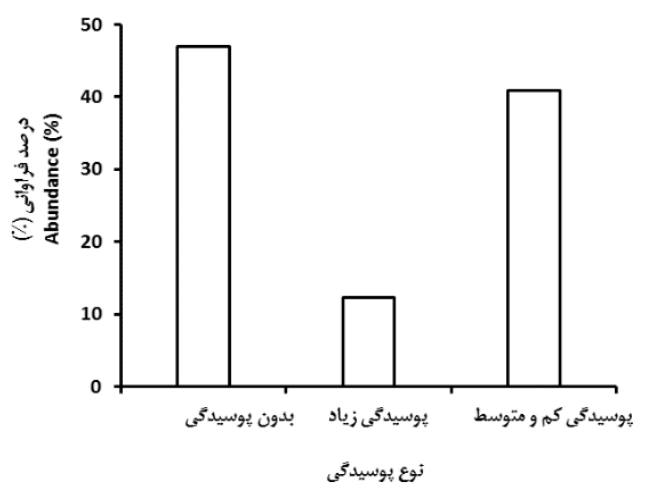

$$
\text { شكلq- نوع يوسيدگى تمام קايههاى نشانهگذارى }
$$

Figure 9. Severity and amount of decay among all marked trees

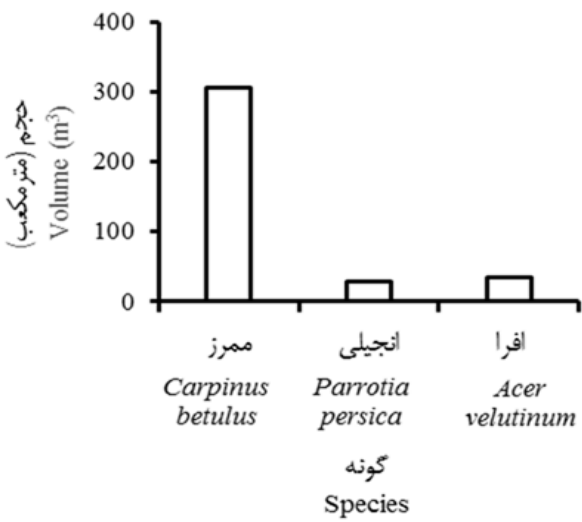

شكل ع- حجم جوب برداشت شده به تفكيك گونه

Figure 4. Volume of marked trees by species

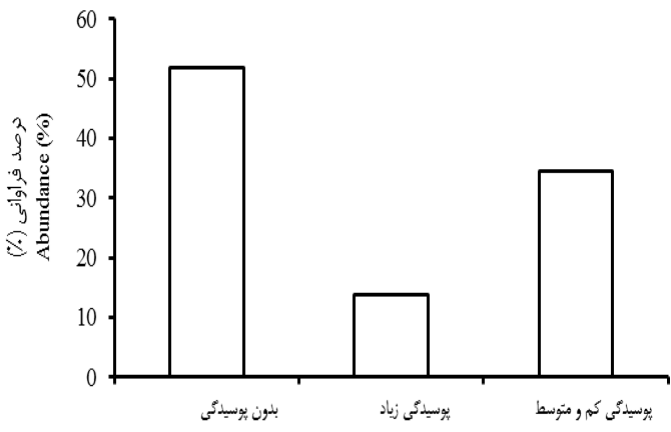

شكل צ- نوع :وسيدگى پايههاى نشانه گذارى شده انجيلى

Figure 6. Severity and amount of decay of marke trees based on species Parrotica persica

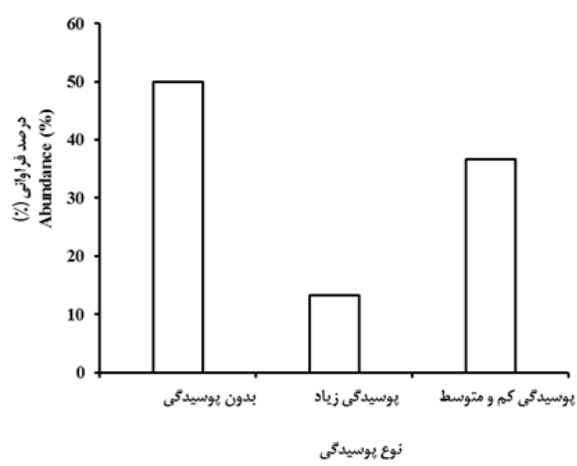

شكل ^- نوع يوسيدگى يايههاى نشانه گذارى شده ممرز

Figure 8. Severity and amount of decay of marked trees based on species Carpinus betulus 
جدول أ- مشخصات تجزيهى الخوى يراكنش پايههاى نشانهگذارى شده در قطعه نمونهى مورد بررسى Table 4. Analysis of distribution pattern for marked trees in the studied compartment

\begin{tabular}{|c|c|c|c|}
\hline $\mathrm{P}$ & Z-score & $\begin{array}{c}\text { متوسط فاصله (متر) } \\
\text { Average of distance (m) }\end{array}$ & $\begin{array}{c}\text { آماره كلارك و ايوانز } \\
\text { Index of Clark and Evans }\end{array}$ \\
\hline$\cdot / \ldots$ & $-\Lambda / V \Delta$ & $I V / D S$ & •/OHV \\
\hline
\end{tabular}

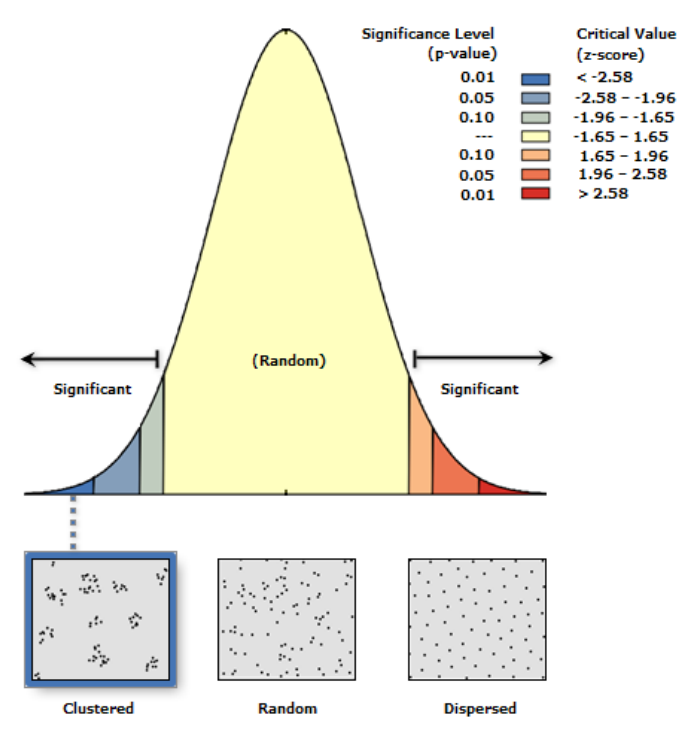

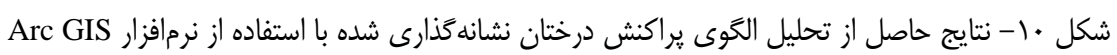
Figure 10. Results of distribution pattern analysis by Arc GIS software

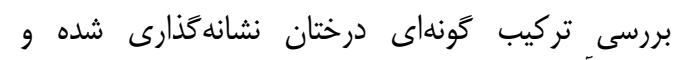

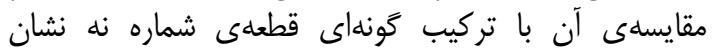

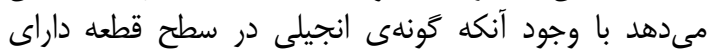

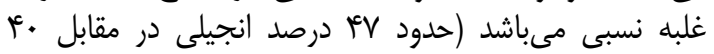

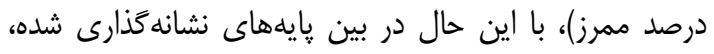

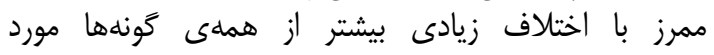

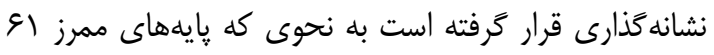

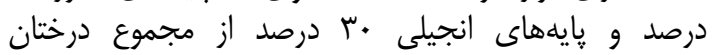

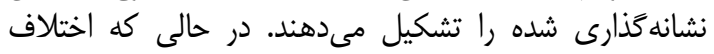

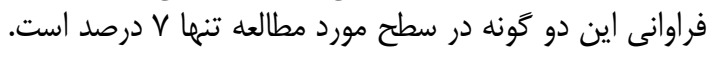

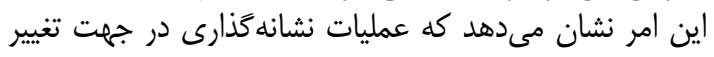

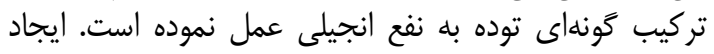

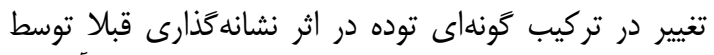

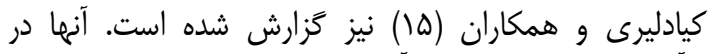

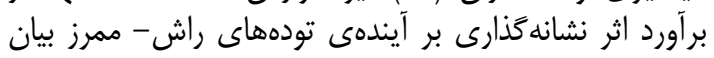

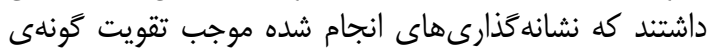

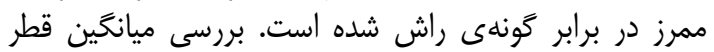

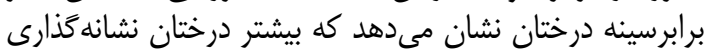

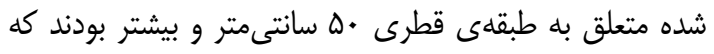

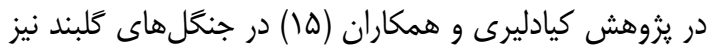

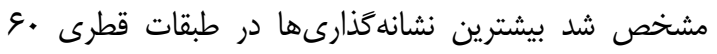

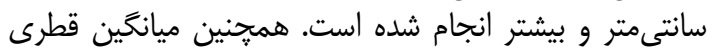

در بررسى الكوى يراكنش درختان نشانه كذارى شده،

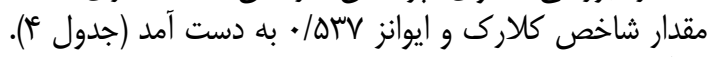

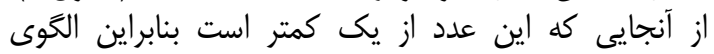

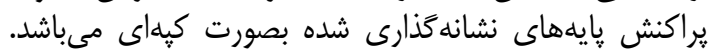

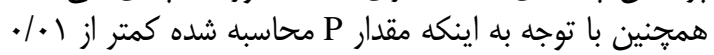

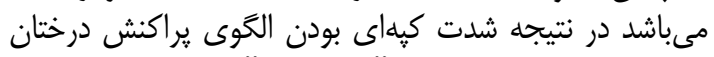

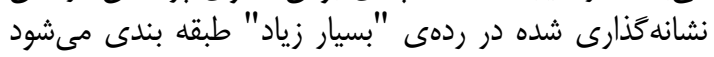

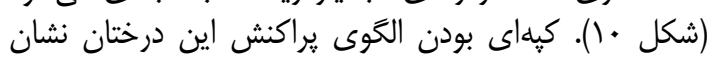

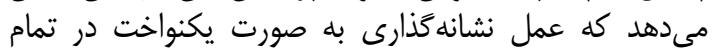

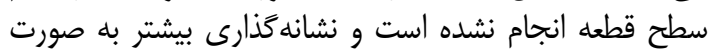

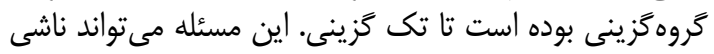

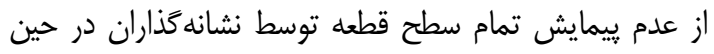

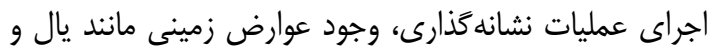

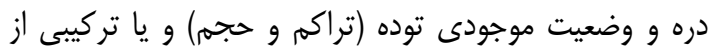

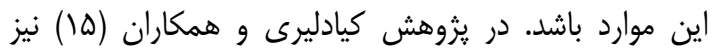

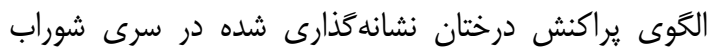

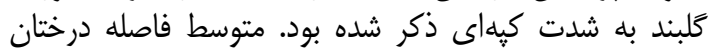

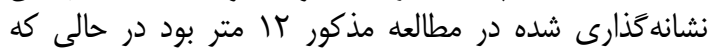

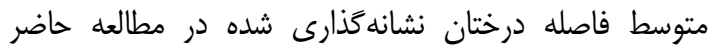

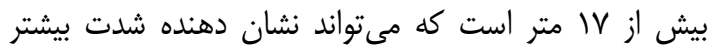
الخوى يراكنش كيهاى درختان نشانه

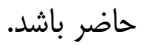


بومسامانهى جنكل دارند و يكى از مشخصههاى ساختارى

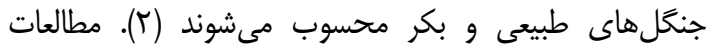

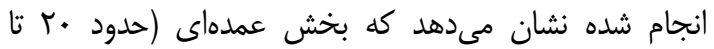

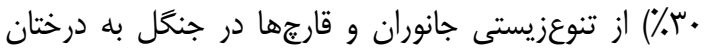

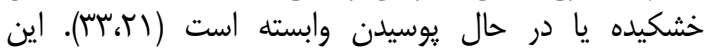

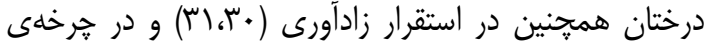

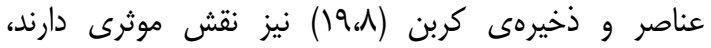

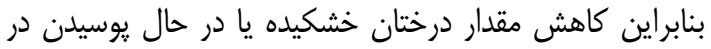

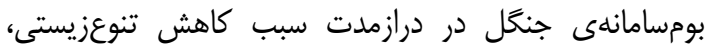

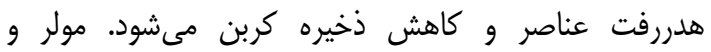

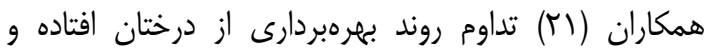

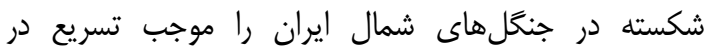

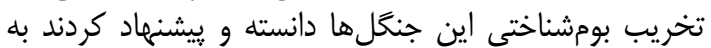

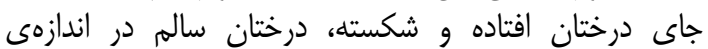

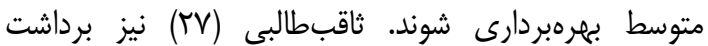

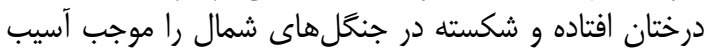

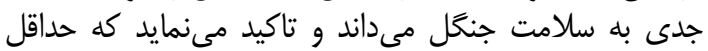

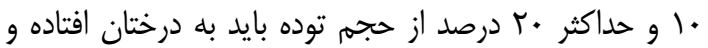
شكسته اختصاص يابد.

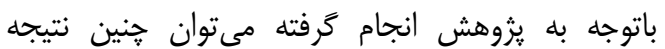

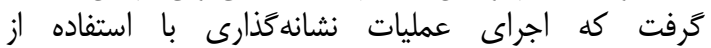

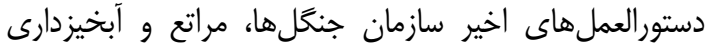

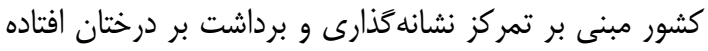

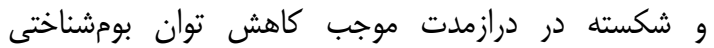

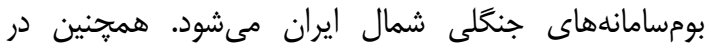

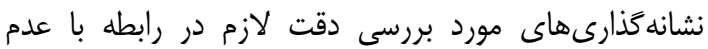

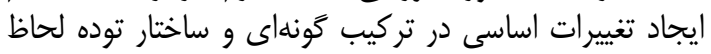

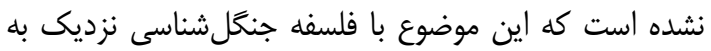

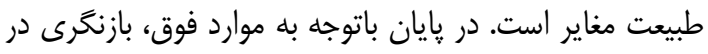
سياست نشانه كذارى درختان افتاده و و شكسته بيشنهاد مى تردد.

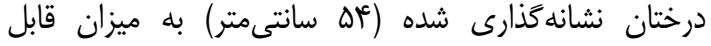

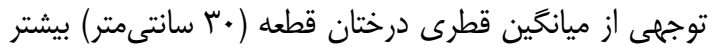

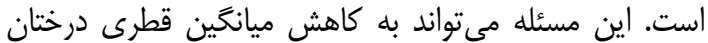
قطعه و جوانتر شدن ساختار سنى توده دئ در آينده منجر شئ شودي.

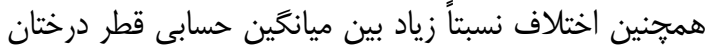

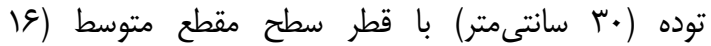

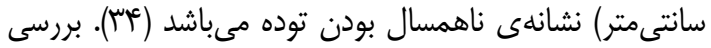

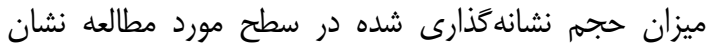

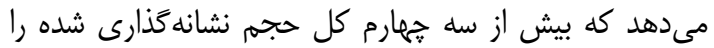

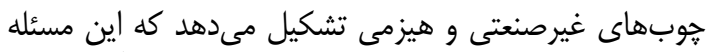

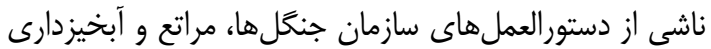

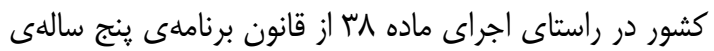

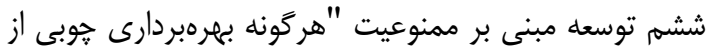

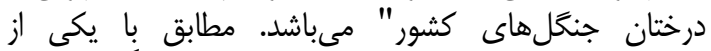

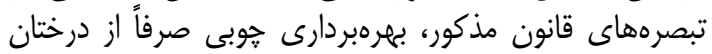

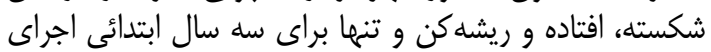

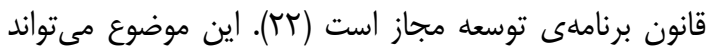

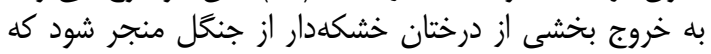

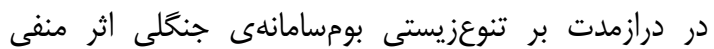

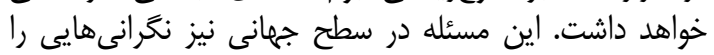

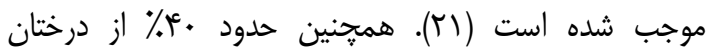

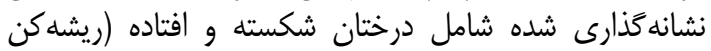

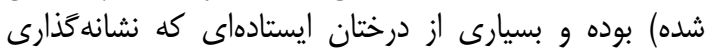

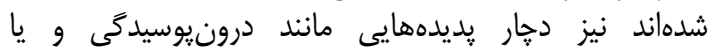

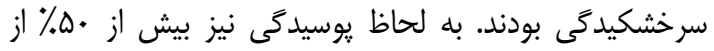

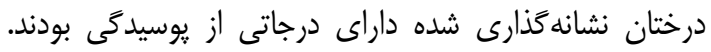

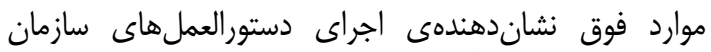

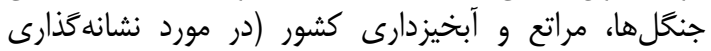
درختان شكسته، افتاده و ريشهكن) توسط نشان أنانه كذاراران است.

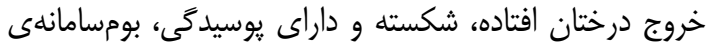

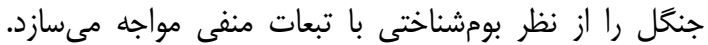
درختان خشكيده و يا در حال يوسيدن اهميت بالايى دراجي در 
منابع

1. Amiri, M., R. Rahmani, K. Sagheb-Talebi and H. Habashi. 2013. Dynamics and structural characteristics of a natural unlogged oriental beech (Fagus orientalis L) stand during a 5years period in Shast Kalate Forest, Northern Iran. Environmental Resources Research, 1(2), 107-129.

2. Amiri, M., R. Rahmani, KH. Sagheb-Talebi and H. Habashi. 2015. Structural characteristics of dead wood in a natural untouched of Fagus orientalis mixed stand forest (Case Study: Shast-Kalateh Forest, Gorgan, Iran). Journal of Wood and Forest Science and Technology, 22(1): 185-205 (In Persian).

3. Charkazi, A., M. Amiri, H. Ravanbakhsh and D. Moghadasi. Examination of quantitative and qualitative characteristics of Cupressus sempervirens var. horizontalis and Pinus brutia in plantation forests in the Ramian, Golestan Province. Journal of Wood and Forest Science and Technology, 23(4): 1-21 (In Persian).

4. Costa, F.W. and M. Magnusson. 2003. Effects of selective logging on the diversity and abundance of flowering and fruiting understory plants in a central Amazonian forest. The Association for Tropical Biology \& Conservation, 35(1): 103-114.

5. Eshaghi-Rad, J. and A. Khanalizadeh. 2014. Quantitative comparison of microhabitats in deciduous forests histories (Case study: Golband- Noshahr). Iranian Journal of forests and Poplar Research, 21(4): 594-605 (In Persian).

6. Eysteinsson, T. 2012. Regeneration after clear-felling and selection-felling in an Icelandic birch forest. Icelandic Agricultural Sciences, 25(1): 37-40.

7. Forest Management plan. 1995. District 1 Dr. Bahramnia forest, watershed 85, Gorgan University of Agricultural Sciences and Natural Resources, $252 \mathrm{pp}$ (In Persian).

8. Harmon, M.E. 2001. Carbon sequestration in forests: addressing the scale question. Journal of Forestry, 99(4): 24-29.

9. Hassanzad Navroodi, I. and S. Hassan Nezhad. 2013. Comparison of quantitative and qualitative characteristics in managed and unmanaged natural forest stands at district Shenrood (Siahkal). Journal of Plants Research, 28(1): 103-115 (In Persian).

10.Hassanzad Navroodi, I. and H. Seyedzadeh. 2013. Effects of shelterwood method on some important forest stands features in Shafarood district nine of Guilan. Iranian Forests Ecology, 1(2): 41-56 (In Persian).

11.Hunter, M.L. 1990. Wild life, forests and forestry: Principle of managing forests for biological diversity. Audubon Field Notes, 24(1): 722-726.

12.Jalilvand, H., M. Niknejad and M. Hejazia. 2015. Evaluating effects of various physiographic factors and distance from road on timber marking using multiple linear regression model. GIS Techniques in Natural Resources, 6(3): 46-59 (In Persian).

13.Kazemi, SH., S.M. Hodjati, A. Falah and K. Barari. 2014. Effect of single selection method on woody and herbaceous plant biodiversity in Khalil-Mahale Forest, Behshahr. Applied ecology, 4(11):15-25. (In Persian)

14.Khanlari, D. 2006. Silviculture and Forestry of Iran (Hyrcanian, Zagros, Arasbaran). Applied Agricultural sciences press, Tehran, 360 pp (In Persian).

15.Kia-Daliri, H., R. Akhavan and I. Anissi. 2011. Timber marking and its impact on forest stand (Case study: Shourab district of Golband region). Iranian Journal of Forest, 1(3): 49-59 (In Persian).

16.Kraus, D., R. Butler, F. Krumm, T. Lachat, T. Larrieu, Y. Mergner, T. Paillet, A. Rydkvist, A. Schuck and S. Winter. 2016. Catalogue of tree microhabitats Reference field list. Integrate+ Technical Paper, $16 \mathrm{pp}$.

17.Malik, A.U., F.W. Bell and Y. Gong. 1997. Regeneration behavior of Competing Plants after clearcutting: implications for vegetation management. Forest Ecology and Management, 95(1):1-10.

18.Marvi Mohadjer, M.R. 2011. Silviculture. University of Tehran Press, 418 pp (In Persian).

19. Merganicova, K. and J. Merganic. 2010. Coarse woody debris carbon stocks in natural spruce forests of Babia hora. Journal of Forest Science, 56(9): 397-405.

20.Mosadegh, A. 2011. Silviculture. University of Tehran press, 490 pp (In Persian).

21.Muller, J., Kh. Sagheb-Talebi and S. Thorn. 2016. Protect Iran's ancient forest from logging. Science, 355(6328): 919-919. 
22.Official newspaper collections. 2017. Available for http://www.dastour.ir/brows/?lid=373941. Accessed $15^{\text {th }}$ September 2018.

23.Pommerening, A. 2002. Approaches to quantifying forest structures. Forestry, 75: 305-324.

24.Rahimiyan, M.S., M. Hassani and H. Kia-Daliri. 2014. Effect of marking on the spatial distribution and structure of beech stands (case study: Safarood-Ramsar). Iranian Journal of Forest and Poplar Research, 22(4): 597-608 (In Persian).

25.Ren-hui, Q., C. Han and Z. Li-Xin. 2006. Effects of selection cutting on the forest structure and species diversity of evergreen broad leaved forest in northern Fujian, Southern China. Forestry Studies in China, 8(1): 16-20.

26.Saab, V. 1999. Importance of spatial Scale to habitat use by breeding birds in riparian forests, a hierarchical analysis. Ecological Applications, 9(1): 135-151.

27. Sagheb-Talebi, Kh. 2017. Dead wood role in forest ecosystem health. Iran Nature, 2(2): 20 25 (In Persian).

28.Sagheb-Talebi, Kh., B. Delfan Abazari and M. Namiranian. 2005. Regeneration process in natural uneven-aged Caspian beech Forests of Iran. Schweizerische Zeitschrift fur Forstwesen, 156(12):477-480.

29.Sagheb-Talebi, Kh., T. Sajedi and M. Pourhashemi. 2014. Forests of Iran, a treasure from the past, a hope for the future. Springer, Berlin, $160 \mathrm{pp}$.

30.Sefidi, K., M.R. Marvi-Mohajer, M. Zobeiri and V. Etemad. 2007. Investigation on dead trees effects on natural regeneration of oriental beech and hornbeam in a mixed beech forest. Iranian Journal of Forest and Poplar Research, 15(4): 365-373 (In Persian).

31.Sefidi, K., Z. Pour-Gholi, Kh. Sagheb-Talebi and F. Keivan-Behjo. 2016. Structural characteristics of canopy gaps in the gap making phase in the evolution of beech stands in the Asalem forests- Guilan. Iranian Forests Ecology, 4(7): 43-50 (In Persian).

32.Silander, J.A. 2001. Temperate forests. Encyclopedia of Biodiversity, 5(1): 607-625.

33.Stokland, J.N., J. Siitonen and B.G. Jonsson. 2012. Biodiversity in deadwood. Cambridge University Press, Cambridge, UK, 524 pp.

34.Taheri Abkenar, K. and B. Pilehvar. 2008. Silviculture. Haghshenas Publication, Rasht, Iran, $300 \mathrm{pp}$ (In Persian).

35.Tavankar, F., J. Mahmoodi and N. Iranparast Badaghi. 2011. The effect of single selection method on tree species diversity in the northern forests of Iran (Case study: Asalem-Nav, Guilan province). Journal of Sciences and Techniques in Natural Resources, 6(1): 27-40 (In Persian). 


\title{
Evaluation of Tree Marking in Persian Ironwood-Hornbeam Stands at Bahramnia Forestry Plan and Its Effect on Stand Structure
}

\author{
Akram Haghighatdoust ${ }^{1}$ and Seyed Mohammad Waez-Mousavi ${ }^{2}$ \\ 1- MSc of Silviculture and Forest Ecology, Faculty of Forest Sciences, Gorgan University of Agricultural Sciences \\ and Natural Resources \\ 2- Assistant Professor at Faculty of Forest Sciences, Gorgan University of Agricultural Sciences and Natural \\ Resources (Corresponding author: waezmousavi@gau.ac.ir) \\ Receive: May 11, $2019 \quad$ Accepted: June 11, 2019
}

\begin{abstract}
Tree marking can have a tremendous effect on structure of forest. This study aimed to assess tree marking operation and to predict its effect on stand structure of Persian ironwood-hornbeam stands at Bahramnia forestry plan, north of Iran. Therefore 98 trees that were marked in compartment 9 in 2017 based on selection system and according to the recent laws of Forests, Range and Watershed Management Organization of Iran were measured before cut. Characteristics such as tree species, diameter, height, volume, decay condition and standing or fallen situation of the trees were recorded. Independent samples t-test was used to compare the mean diameter, height and volume between marked trees and stand trees. To obtain the mean diameter and height of the stand trees 16 circular sample plots with the area of 1000 square meters were considered in which the diameter and height of all trees were recorded. The results showed that the majority of marked trees, based on both tree numbers and volume, belong to hornbeam species while the dominant tree species of the compartment is Persian ironwood tree. This result shows that the marking operation acted to change the species composition of the stand in favor of Persian ironwood species. In comparison of the mean diameter, height and volume between marked trees with stand trees a significant difference were observed in terms of diameter and volume, but in case of trees height no significant difference was found. Studying the distribution pattern of marked trees indicated a cluster distribution pattern. Totally, it can be concluded that the tree marking operation according to the recent laws of Forests, Range and Watershed Management Organization, which emphasize on marking and cutting of fallen and broken and decaying trees, in long term will result in loss of dead and decaying trees in forest ecosystem and will decrease ecological capacity of these forest ecosystems.
\end{abstract}

Keywords: Silviculture, Structure, Selection System, Microhabitat, Distribution Pattern 